\title{
FUENTES DE TENSIÓN EN EL EJERCICIO PROFESIONAL DEL PROFESOR DE CIENCIAS
}

\author{
LATORRE IATORRE, A., FORTES DEL VALLE, M C. y SERRA DESFILIS, E. \\ Departamento de Psicología Evolutiva y de la Educación. Universitat de València.
}

\begin{abstract}
SUMMARY
The main aim of this article is to present a conceptualization of the causes of stress in teachers, based on a review of recent investigation concerning this subject and stating the importance of the working conditions, interpersonal relations, personal development, initial and continual formation and relations with society as causes of stress. A mention is made of how small the incidence of stress is on science teachers compared to their companions of other subjects.
\end{abstract}

\section{INTRODUCCIÓN}

Es de uso común el considerar que los profesoress están expuestos a los efectos psicológicos de la tensión en el ejercicio de su profesión. Cada vez se hace más evidente la existencia de un creciente malestar entre los enseñantes, malestar que se traduce en absentismo, abandono del puesto de trabajo y enfermedades nerviosas. Esta situación ya fue advertida por Mandra en 1977 al afirmar:

«Hace veinticinco años las ausencias de larga duración correspondían en un $75 \%$ a enfermedades respiratorias u orgánicas; hoy esta proporción se halla representada por las afecciones de orden mental (depresiones nerviosas, neurosis, psicosis) que precisan una readaptación.

»Por añadidura, los enseñantes que se "hundían" hace todavía diez o quince afios se encontraban al final de su carrera, mientras que ahora el fenomeno afecti a un número importante de los menores de 30 años.»

Estos datos han sido contrastados en múltiples investigaciones efectuadas en diferentes contextos sociologicos con resultados similares. Así, en 1983 la OIT informaba que «el porcentaje de bajas laborales por causas psíquicas o psicosomáticas aumentaba rápidamente entre los colectivos docentes". Hargreaves (1978) denomina a esta situación del agotamiento mental y emocional progresivos, «la apatía clínica del profesor gastado». Amiel (1985), en Francia, destaca los aspectos tersionales ansiosos o de fatiga entre los docentes, señalando «en lugar central la frustración y la depresión, con manifestaciones como apatía, desinterés, dificultad de concentración, desvalorización personal, desesperación... jun- to a insomnio, ansiedad u manifestaciones psicosomáticas de tipo muscular, digestivo, cardiorespiratorio. Mandra (I984) comprobó cómo los afectados por enfermedades mentales entre los enseñantes tripiclaban a los no docentes en el régimen general de la Seguridad Social en Francia. Esteve (1988) de la universidad de Málaga, hace años que viene publicando datos sobre la incidencia de este hecho en la población española, llegando a diseñar un modelo de intervención.

El estado psíquico del profesor influye en su conducta, generalmente la tensión desencadenada en el ejercicio de la profesión docente origina ideas de ansiedad, desánimo, temor, irritabilidad, con respecto a los estudiantes, compañeros; padres de alumnos... e indudablemente repercute en el rendimiento del profesor.

En este trabajo hemos querido recopilar parte de la información existente sobre el tema y analizar las causas que pueden originar tensión en el colectivo de profesores de Ciencias.

\section{FUENTES DE TENSIÓN EN EL EJERCI- CIO PROFESIONAL DEL PROFESOR DE CIENCIAS}

En general las fuentes de tensión en el trabajo del profesor de Ciencias no tienen por qué diferir demasiado 
del resto de profesores que trabajan otras áreas, pero, si se quiere dar a esta materia un enfoque experimental, surgen sin duda unos focos de tensión muy específicos.

El estudio de Mandra (1984) sobre «Causas de inadaptación y desadaptación de Ios enseñantes franceses y dispositivos de ayuda puestos en marcha por el Ministerio de Educación Nacional", encontró que había entre los profesores de secundaria un mayor número de la especialidad de letras que disfrutaban de permisos de inactividad de larga duración. Las razones de estas diferencias significativas entre ciencias y letras no están claras, aunque se argumenta que los de ciencias tienen más salidas laborales y se encuentran menos abocidos que los de letras a la enseñanza. También se apunta que las materias de ciencias causan menos tensión porque dan mayor prestigio ante los padres, los alumnos y los demás compañeros, ofrecen un menor margen a la interpretación y a la valoración ideológica; y los aprendiziajes adquiridos permiten en mayor medida una evaluación más mecánica e impersonat. Sin embargo, consideramos que la mayor parte de estos análisis no son extrapolables a la EGB, porque las salidas profesionales son las mismas, puesto que todos, ciencias y letras, parten de una misma titulación general y las fuentes de tensión están más determinadas por el modo en que se quiere trabajar que por la materia en sí.

Las distintas investigaciones realizadas sobre las tensiones que sufre el profesor y la repercusión en su rendimiento taboral han explorado fundamentalmente los siguientes focos de tensión:

2.1. Condiciones de trabajo (horario, número de alumnos por clase, locales, material...)

2.2. Relaciones interpersonales (con los alumnos, los padres, los colegas, la dirección, la inspección).

2.3. El desarrollo personal (la forma en que los enseñantes viven la profesión y se desenvuelven en ella).

\subsection{La formación inicial y continuada.}

2.5. Las relaciones con la sociedad.

\subsection{Condiciones de trabajo}

Las condiciones de trabajo: horarios, número de alumnos, locales y materiales, pueden aparecer como fuentes potenciales de estres en el profesor de ciencias.

Con respecto al horario, el profesor de Ciclo Inicial y Medio que quiere realizar experimentación puede encontrar una fuente de tensión en asignar cl tiempo adecuado a esta actividad sin detrimento de otras materias básicas en estos ciclos.

A pesar del descenso de natalidad que estamos viviendo, la mayoría de las aulas de EGB siguen teniendo una ratio demasiado amplia para la realización de clases prácti- cas-experimentales, en las que el número ideal de niños debe oscilar entre 15-20; pero el criterio varía según la actividad y el aprendizaje previo que tengan los estudiantes en estas actividades. Para prevenir la tensión de trabajar con un número inadecuado y que las actividades prácticas constituyan una fuente excesiva de tensión, cl profesor de Básica que quiere experimentar se ve obligado a ensayar distintas alternativas que le permitan superar csta dificultad: dividir el grupo-clase para experimentación si el número de profesores del centro lo permite, realizar primero algunas actividades más tecnológicas con un número reducido de alumnos -que luego serán los jefes de equipo- y contar con ellos como apoyo para no verse desbordado en el aprendizaje del manejo de algunos aparatos, etc.

El profesor de Ciencias de Ciclo Superior puede encontrar una fuente de tensión en el hecho de no disponer de un auia de uso exclusivo en la que crear un cierto "clima» teniendo que rotar por las distintas clases. Tambićn es una fuente de tensión el no disponer de un aula-laboratorio que reúna unas condiciones mínimas de espacio y equipamiento. Y cuando se dispone de ella, las tensiones vienen por dedicarle el ticmpo necesario para tenerla adecuadamente organizada y sacarle el máximo rendimiento. La gestión, organización y conservación del material de ciencias puede ser otra fuente de tensión para el profesor.

\subsection{Relaciones interpersonales}

Constituyen el clemento más problemático de la actividad docente, sicndo los focos más productores de estrés: las relaciones con los estudiantes, con los compañeros docentes, con las familias, con la direccion y con la inspección.

\section{Con los alumnos:}

Vera (1988) señala que las relaciones con los alumnos representan uno de los aspectos de la profesión que mayor satisfacción puede aportar al profesor, por eso mismo constituye también una de las mayores fuentes potenciales de malestar. Esto es lógico porque los parámetros básicos empleados en la valoración de su trabajo son : su capacidad para hacer progresar a los alumnos y su aptitud para logrario sin que se altere el orden en la clase. Hl profesor tiene que impartir sus cnscñanzas a un grupo de alumnos normalmente heterogenco y como además el número de éstos suele ser grande, en muchos casos el profesor suele encontrar dificultades para interpretar a su interlocutor y acaba, con frecuencia, optando por dirigirse a un alumno medio inexistente. Este es un riesgo especial para los profesores del área de matemáticas y ciencias, ya que, como señala el informe Cockcroft (1982), son materias generalmente jerarcuizadas, por lo que la comprensión de una cuestión depende en buena parte de la comprensión previa de cuestiones anteriores, y existe una gran diferencia en el rendimiento y ritmos de aprendizaje entre unos alumnos y otros -en la investigación realizada por este matemático se encontraron diferencias de hasta siete años en relación con el 
momento en que se logra la comprensión de aigunas cuestiones.

La dificultad para compatibilizar el logro de los objetivos propuestos con el establecimiento de un clima positivo de clase, hace que la conducta del profesor fluctúe entre la necesidad de hacer imperar unas normas mínimas que regulen y ordenen el trabajo en la clase y cl trato cordial que quierc tener con sus alumnos. Puede asumir el papel de « líder impuesto» (ejerciendo el poder por las prerrogativas que le confiere su posición oficial) o de «líder natural» (en caso de merecer la confianza y el reconocimiento de sus alumnos), y puede encontrarse a veces basculando entre ambas posiciones.

Abraham et al. (1986), en su obra El enseñante es también una persona, reflexiona en torno a la cuestión de que el profesor es alguien que precisa encontrar en su trabajo un clima de aceptación que le estimule a buscar métodos cada vez mejores. Necesita el apoyo, la comprensión y la consideración de toda la comunidad escolar y social; pero fundamentalmente precisa saberse estimado y valorado por sus propios alumnos ya que es con ellos con quien más tiempo convive.

El modelo de Esteve (1987) que representa los elementos que intervienen en el malestar docente permite describir cuatro consecuencias posibles del malestar en la relación profesor-alumno.

Primer caso: Son los profesores que habiendo recibido una formación inicial adecuada o inadecuada logran a través del aprendizaje adquirido por ensayo y error, en los primeros años de ejercicio docente, desarrollar comportamientos adecuados; tienen seguridad suficiente ch sí mismos y se implican en el trabajo diario. Las fuentes del malestar les hacen trabajar con la tensión propia de las tareas que, siendo muchas y diversificadas, les exigen un sobreesfuerzo del que obtienen un nivel notable de logros. Se sitúan en niveles de estrés positivo y beneficioso para una consecución eficaz de las actividades profesionales (cuestrés), lo que les hace persistir en el esfuerzo (anástasis) con un aito grado de motivación y satisfacción. Éste es el grupo de los profesores que logran una autorrealización en su trabajo y que luchan por mejorarlo día a día. Es posible según nuestra percepción que los profesores de ciencias que trabajan con experimentación en sus clases, pertenezcan a este primer grupo.

Segundo caso: Lo representan aquellos profesores que recibieron una formación inicial inadecuada y que además no han adquirido en la práctica un nivel suficiente de aprendizaje por ensayo y error. No han conseguido desarroltar pautas de actuación acordes con las demandas del momento y se muestran faltos de confianza en sí mismos. Es un grupo que recurre a prácticas docentes y modelos de intervención en los que no se implica personalmente. Disminuye su esfuerzo en el trabajo y también decrece su motivación y el grado de satisfacción obtenido. Su enscñanza se caracterizará por la inhibición ante los acontccimientos importantes, por ia rutina y la burocratización. Un cjemplo de este caso en profesores de ciencias son los "prácticos» de nuestra tipología.
Tercer caso: Es similar al anterior, pero el profesor se debate contradictoriamentc entre las actitudes superadoras y las dimisionistas. Su práctica va a estar presidida por la discontinuidad en cl esfuerzo y la contradicción. Ante la falta de recursos apropiados y cl desbordamiento a que se ven sometidos, sus respuestas están presididas por momentos de esfuerzo intenso seguidos de decepción y abandono.

Cuarto caso: Es el grupo más preocupante desde una perspectiva clínica, está constituido por aquellos profesores que no habicndo recibido una formación adecuada tampoco han sabido solucionas con la experiencia los problemas encontrados en las situaciones educativas. A pesar de ello, mantienen un grado de implicación personal muy elevado que les impide despreocuparse de su trabajo. Se esfuerzan mucho en la ejecución de las tareas y están fuertemente motivados, pero van a sentirse muy insatisfechos ante la faita de recompensas de sus desvelos, lo que puede llevar a quebrantar su salud debido a una fuerte depreciación del yo, un estado permanente de ansiedad y la aparición de, en ocasiones, neurosis reactivas o depresiones.

\section{Con los compañeros:}

L.a dificultad para establecer buenas relaciones con los compañeros puede ser una fuente de tensión en el profesor de Ciencias, especialmente por las necesidades de intercambio que necesita. A veces la inseguridad impide que realice preguntas necesarias y otras, algunas barreras cognitivas le condicionan negativamente a prestar a otros compañeros la ayuda que necesitan, cuando para alcanzar un buen nivel profesional es necesario en esta disciplina, como en otras, trabajar en equipo.

«Normalmente la gente no busca ayuda para los episodios ocasionales del estrés, aun cuando tales episodios puedan producir un trastorno importante y un deterioro en el funcionamiento" (I azarus y Folkman 1986).

Tradicionalmente cada profesor ha resuelto individualmente, como ha podido, las dificultades surgidas de un trabajo solitario. Por ello no es de extrañar la aparición de la sensación de desamparo y soledad ante la clase (Martínez 1984).

La falta de entrenamiento en el trabajo cooperativo con compañeros a los que uno puede abrirse para intercambiar métodos de trabajo, opiniones, vivencias, etc., se pone de manifiesto, por ejemplo, en la resistencia que oponen en asumir experiencias de autoconfrontación, las cuales provocan miedo a ser evaluado, nerviosismo y la aparición de fuertes autocríticas (Vilkar Angulo 1984).

\section{Con los padres:}

El docente no siempre encaja bien que no se acepte todo lo que dice. Se sentirá agredido por lo que los padres 
quieran imponerle o incomprendido si se le critica (Gosselin 1984).

Aquellos profesores que más se esfuerzan, en ocasiones por ir contracorriente, por emplear metodologías innovadoras, tropiezan con la incomprensión de algunos padres preocupados por la posibilidad de que tales innovaciones puedan ocasionar retraso en el número de conocimientos que han adquirido sus hijos (Elejabeitia 1983).

Wilson (1980) señala que los profesores consideran que los conflictos en las relaciones personales dentro de la institución y más concretamente en las relaciones con los padres son un obstáculo para la tarea de educar, pero así como los problemas en la relación con los alumnos son sistemáticamente mencionados en las investigaciones sobre fuentes de tensión del profesor, no ocurre lo mismo en las relaciones con los padres.

En el trabajo de Joung (1980) este elemento no aparece entre las veinte preocupaciones más importantes de los profesores. No aparece tampoco entre los trabajos revisados por Coates y Thorensen (1976) que se referian a los profesores veteranos. Lo mismo sucede con otras investigaciones (Kyriacou y Sutcliffe 1978, Chinchon y Koff 1978 , Golladay y Noel 1978 y Pettegrew y Wolf 1982).

I as relaciones con los padres sí se mencionan en las investigaciones dedicadas a los profesores en sus primeros años de ejercicio profesional, como la realizada in el contexto europeo por Breuse (1984)

Un área de tensión importante para el profesor de ciencias en relación con los alumnos y sus familias son las salidas extraescolares, imprescindibles para trabajar adecuadamente algunos temas y que representan una dificultad organizativa y un área de peligro muy considerable, ya que lat ratio 1/35 obliga en muchos casos a realizarlas con un alto número de niños, y, en este punto, como en el de la experimentación, pensamos que debe primar siempre la seguridad por encima de cualquier otro criterio, con la consiguiente frustración que eso puede producir en ocasiones.

\section{Con la dirección y con la inspección:}

El director escolar precisa ser una persona muy preparadia.

«Las condiciones pedagógicas constituyen el núcleo medular de la personalidad del director, pero no debe olvidarse que también se requieren otras aptitudes para el ejercicio eficaz de la función directiva como son: capacidad de gestión y organización, dotes de relación social, conocimientos gerenciales en materia de personal, en cuestiones económicas, etc." (Latorre 1984).

Para ejercer con eficacia la función directiva (función de mecanismo de enlace), es imprescindible que la persona que desempeñe el rol de director reúna una serie de aptitudes humanas:
-Personalidad centrada.

-Equilibrio personal.

-Capacidad para saber escuchar a los demás.

-Capacidad para discernir e investigar la certeza de la información recibida.

-Capacidad de gestión y organización.

-Predisposición para orientar, informar y dirigir.

Cuando en un centro se encuentra un director con estas características la vida escolar se dinamiza y las tensiones se suavizan, pero, dada la forma en que se accede a la dirección y la dedicación que el cargo requiere, no siempre llevan esta gestión los más capacitados, sino los que quieren y pueden realizarla.

\section{Encontramos en nuestros colegios:}

-Directores dedicados (producir o morir) $(9,1)$.

-Directores relacionados (laiser faire) $(1,9)$.

-Directores burócratas (mínimo esfuerzo, mínimo rendimiento) $(1,1)$.

-Directores integrados (director ideal) $(9,9)$.

Con las coordenadas representadas junto a cada tipo podríamos establecer en una rejilla, las combinaciones posibles de esta tipología.

Por lo tanto, las tensioncs que se produzcan en la relación profesor-director, dependerán fundamentalmente de la interacción entre ambos tipos.

\section{Con la inspección:}

Según Vera (1988), la falta de apoyo institucional es principalmente preocupante en el caso de los profesores que empiezan, porque desbordados por los problemas corren el peligro, tras un período de intentos de supera. ción por acabar inhibiéndose y acomodándose a la situación establecida.

Cerca del $70 \%$ de los comentarios relativos a la inspección se orientan en el sentido de una ausencia total de contactos fructíferos con ella. Los consejos pedagógicos son escasos y la inspección es considerada como un órgano de control y no de formación, posiblemente porque su función sólo csté contemplada a nivel organizativo y administrativo. Además según la investigación. de Breuse (1984) la inspección era sobre todo percibida como representante de las ideas de una persona y no de una estrategia educativa de conjunto.

Creemos que, en nuestro país, la inspección no es en la actualidad una fuente de tensión para los profesores y sí, en cambio, las nuevas disposiciones administrativas, no siempre adecuadas desde el punto de vista del maestro y no siempre entendidas por este colectivo. 


\subsection{Desarrollo personal}

En relación con punto de si contribuye la profesión docente al desarrolio personal, el estudio de Breuse (1984) encontró mayor intensidad en las opiniones negativas que en las positivas. Hay profesores felices que consideran que su profesión es enriquecedora y creativa, pero hay un gran número que admite haber perdido el entusiasmo; otros manifiestan agotamiento nervioso, especialmente los que trabajan con niños más pequeños; un buen número reprochan a su profesión no dejarles un momento de respiro; y otros tienen la impresión de no poder expresarse, de no ser ellos mismos. Se advierte en ellos un sentimiento de frustración y desánimo y comoes la evaluación cognitiva el determinante básico de la emoción, ésta se presentará de una manera negativa .

«El individuo está en función de la situación, pero también y más importante es que (...) la situación está en función del individuo a través de: $a$ ) la construcción cognitiva que éste haga de ella, y $b$ ) la selección activa y modificación que lleve a cabo." (Ekehammar 1974). (Citado por Lazarus y Folkman, 1986).

Vera (1988) cree que existe evidencia suficiente para afirmar que la consecuencia fundamental derivada de las fuentes de malestar entre los docentes no son reacciones patológicas o desequilibrios patológicos graves, sino más bien sentimientos de malestar, frustración, euestrés, y la aparición de mecanismos de inhibición y rutina, y solo un reducido número de profesores diffciles de estimar cuantitativamente por la variedad de instrumentos de recolección e interpretación de datos empleados ven afectada su salud física y mental por los efectos de las fuentes de malestar. Influyen en ese malestar por un lado la quizás deficiente formación inicial y por el otro las dificultades que para una adecuada formación permanente encuentran los profesores en ejercicio. Hecho que posibilitaría el adecuado desarrollo personal del docente.

\section{4. $\mathrm{Kl}$ profesor de ciencias y la sociedad}

La sociedad es también otra fuente de tensión para el profesor de ciencias, porque las expectativas sociales han aumentado extraordinariamente. Mientras que a comienzos de siglo se esperaba que la escueia instruyese mínimamente en instrumentos como el cálculo y la escritura, actualmente los contenidos curriculares abarcan la práctica totalidad de muchos aspectos de la reali. dad y cada día son más los campos que pugnan por ser incorporados (educación para la salud, educación medio-ambiental, educación vial....).

Ha habido además una complejidad creciente en las tareas educativas que han adquirido un carácter más técnico, con la consiguiente necesidad de una mejor preparación científica y tecnológica (sobre todo si el área de conocimientos en la que se trabaja son las ciencias). Sin embargo la estructura organizativa del sistema escolar no ba sufrido la transformación que se necesitaba, por lo que, para satisfacer las demandas sociales, los profesores deseosos de atenderlas han visto aumentar sus funciones llegando a un estado de sobresaturación, ya que elementos de apoyo al profesor como auxiliares administrativos, ayudantes de raboratorio... no se contemplan en los niveles básicos.

Por otra parte, la nueva forma de entender los conceptos de enseñanza-aprendizaje plantea como objetivos el desarrolio intelectual, estimular la comprensión, desarrollar el espíriritu crítico, Ios hábitos de trabajo, técnicas de estudio... Las nuevas funciones que debe realizar el profesor requieren un mayor protagonismo del alumno en clase y esta enseñanza basada en una relación más participativa necesita unas dotes organizativas muy importantes, además de un incremento considerable en las tareas de preparación y diseño.

«En contraste con el profesor clásico, el profesor de hoy es (debe ser) muchas más cosas (...) parte de un equipo de especialistas que investiga la mejor forma de enseñar los contenidos de un área de conocimientos (departamentos), micmbro de un grupo de profesores responsables de la enseñanza de los alumnos de un determinado ciclo, un animador de grupos de trabajo escolar e individualizador en la asignación de materiales y situaciones de aprendizaje en función de las necesidades de cada alumno, un micmbro de la comunidad escolar participativa en la que están presentes con sus intereses y sus puntos de vista todos los estamentos implicados en la educación de los alumnos, un colaborador de los padres, etc. (González-Anleo 1985).

Pero puede ser que el principal desánimo del profesor proceda de la desvalorización del oficio y de la pérdida del estatus social en otros tiempos quizás envidiable (a nivel de prestigio, aunque nunca a nivel cconómico).

Aigunos se preguntan sobre su rol, tal vex sobre su utilidad en la sociedad; Ios hay que lamentan que esta profesión, ante todo vocacional, sea una práctica rutinaria para muchos. No faltan las quejas por la incomprensión de que son víctimas en el marco de la institución escolar y por la discriminación reinante entre los enseñantes de diferentes niveles.

Estas evaluaciones tan negativas son preocupantes y productoras de desadaptaciones porque las personas consiguen mejores resultados adaptativos cuando creen que van a recibir apoyo social cada vez que lo necesitan (Lazarus y Folkman 1986).

\section{CONCLUSIONES}

Siguiendo a Serra (1989) podemos concluir que el camino profesional de un profesor está lleno de sucesos vitales, productores de estrés y productores de cambios, y quizás la sociedad tenga que intervenir más de lo que lo ha hecho hasta el momento para que esos cambios adquieran la direccionalidad correcta. En caso contrario, sería conveniente aprender a cmplear estrategias adecuadas de afrontamiento como forma de afrontar las situaciones de estrés productoras de tensión en el profesor. 
Además, como ya se ha expuesto en el contenido del trabajo, «las investigaciones sobre el agotamiento del profesor nos lo muestran como un profesional sobrepasado, al que, por la acumulación de responsabilidades y expectativas, desproporcionadas para el tiempo y los medios de que dispone, se le condiciona a no hacer adecuadamente su trabajo» (Esteve 1987). Este hecho otra causa capaz de producir estrés y tensión en el ejercicio de la profesión docente.

A la vista de esta problemática tan compleja, es evidente que no deben realizarse análisis simplistas valorando el nivel de calidad con que los profesores rabajan las ciencias en la educación primaria. La situación educativa incluye una amplia gama de variables que incremen- tan la complejidad del proceso de aprendizaje. Todo profesor que pretenda optimizar al máximo tanto las múltiples variables como su interacción estará sometido a situaciones de tensión, que en el área de ciencias pueden verse incrementadas cuando se pretende impartir Ias clases de forma experimental.

Finalmente, en el cuadro que presentamos a continuación, realizado por Serra y Gomez (1989) recogemos un esquema explicativo de las alteraciones comportamentales en el personal docente, así como las fases de un Plan de Intervención y Prevención elaborado por los autores, que podría servir como síntesis y modelo preventivo de afrontamiento ante la cada vez más frecuente tensión exixtente en el ejercicio profesional del profesor.

Cuadro I

Esquema explicativo de los procesos

I

Conflictividad propia de la profesión en sí y por su coyuntura actual... Ambiguedad en el rol, conflicto de rol y estrés y rol docente. Bajas tasas de reconocimiento/reforzamiento. Descontrol de contingenci.ss...

Ratios elevadas y hetcrogéneas... Alumnos y grupos conflictivos. Deficiencias en medios, en formación del docente, programas, etc.
II

Conflicto de oxigen no profesiona.
III

Problemas concretos, conflictos, enfrentamientos, fatiga acumulada.
IV

Modelos deficitarios de descanso, reforzamiento, realizacion, relaciones sociales.

\section{Carencias personales en habilidades y tecricas per-
sonales y psicologicas para enfrentar los conflictos. sonales y psicologicas para enfrentar los conflictos.}

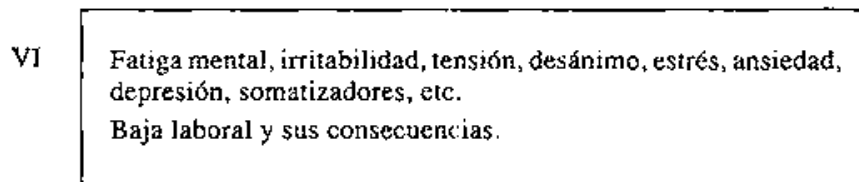

Modelo explicativo de las alteraciones comportamentales en personal docente. E. Serra y L. Gómez (1988).

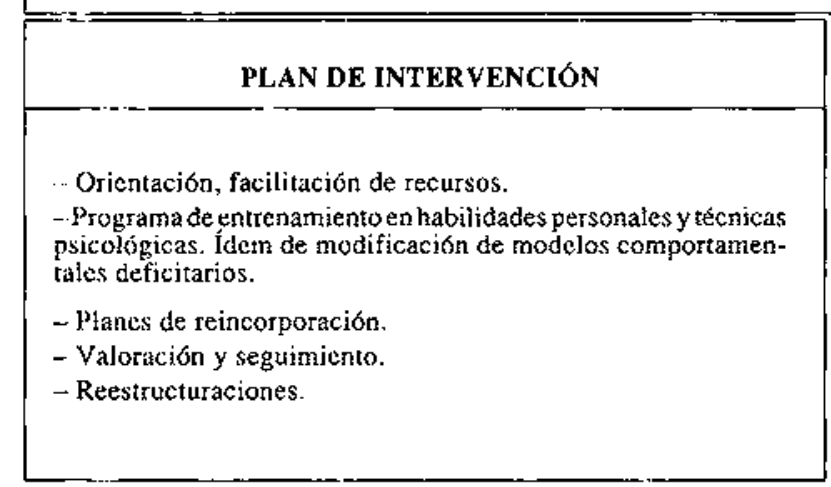




\section{REFERENCIAS BIBLIOGRÁFICAS}

ABRAHAM, A., et al., 1986. El enseñante es también una persona. (Gedisa: Barcelona). (Trad. de L'enseignant est une persone. París. FSF. 1984).

AMIFI, R., 1985. Enteprise santé. (Maloine: Paris).

BREUSF, E., 1984. L'enseignant debutant, Recherche en Education. 29, pp. 110-125.

BREUSSE, E., 1986. Formación de los docentes centrada en la persona, en Abraham et al., obra citada.

COATES,T.J. y THORENSEN,C.E., 1976. Teacher anxiety: A review with recommendations, Review of Educational Research, 46(2), pp. 159-184.

CROCKCROFT, 1982. Comisión para analizar los problemas en la euseñanza de las matemáticas, en Beltrán, Psicología Educacional, Madrid. Fudema. 1987.

CHINCHON,D. J. y KOFF, R.H., 1978. The teaching events stress inventory. Paper presented in the annual meeting of the American Educational Research Association. Toronto.

LKEHAMMAR, B., 1974. Interactionism in personality from a historical perspective, Psichological Bulletin, 81, pp. 10261048 .

ELEJABEITIA, D. de, 1983. F1 macstro. Educación y Socie. dad, pp. 147-166.

ESTEVE, J. M., 1987. El malestar docente. (Laia. Barcclona).

ESTEVE, J. M., 1988. El estrés de los profesores. Propuestas de intervención, en Perspectivas y problemas de la función docente. (Narcea: Madrid).

GOLLADAX, M. y NOEL, J., 1978. The condition of Education. (National Institut of Education: Washington).

GOMEZ, A. y SERRA, E., 1989. Sobre la salud mental de los profesores, Cuadernos de Pedagogía, 175, pp. 60-65.

GONZÁl.EZ,ANI.EO, J., 1985. El sistema educativo español. (Instituto de estudios cconómicos: Madrid).

GOSSELIN, M. F., 1984. Ensayo de identificación de las fuentes de tensión del cnseñante en su trabajo profesional. en Esteve, J., 1987. Profesores en conflicto. (Narcea: Madrid).

HARGREAVES, D., 1978. Las relaciones interpersonales en la educación. (Narcea: Madrid).
JOUNG, T., 1980. Teacher stress : One school district's approach, Action in Teachers Education, 2 (4), pp. 37-40.

KYRIACON, C. y SUTCHIFFF, J., 1978. Teacher stress: Prevalence, sources and symptoms, British Joumal of lidu. cational Psychology, 48. pp. 159-167.

L.ATORRE, A., 1984. Función directiva de Centros Docentes. Tesis de Licenciatura. Universidad de Barcelona.

LATORRF, A. y FORTES, M. C., 1988. Psicología y aprendizajes escolares: Psicopedagogia del área de Ciencias Naturales en $E G B$. Barcelona.

LATORRE, A. y FORTES, M. C. 1989. El maestro investigador: Humanista o técnico, Cuadernos Critic'os de Educación, Valencia, 6, pp. 101-123.

LAZARUS, R. y FOLKMAN, S., 1986. Estrés y procesos cognitivos. (Martinez Roca: Barcelona).

MANDRA, R., 1977. Au-delà du malaise, L'education, 10, pp. 3-77.

MANDRA, R., 1984. Causas de inadaptación y desadaptación de los enseñantes franceses y dispositivo de ayuda puesto en marcha por el Ministerio de Educación nacional, en Esteve, J., Profesores en conflicto. (Naxcea: Madrid).

MARTINEZ, A., 1984. El perfeccionamiento de la función didáctica como vía de disminución de tensiones en el docente, en Esteve, J., obra citada.

PETTEGREW, I. S y WOLF, G. F..1982. Validating measures of tcacher stress, American Educational Research Journal, 19, pp. 373-396.

SERRA, E., GONZÁLFZ, A. y OLLER, A., 1989. Desarrollo adulto: Sucesos evolutivos alolargo de la vida. (Grupo Editor Universitario: Barcelona).

VERA, I., 1988. La crisis de la función docente (Promolibro: Valencia).

VILLAR ANGULO, L, 1984. Resistencias a la autoconfrontación en el entrenamiento de los profesores, en Esteve, J., obra citada.

WILSON, C. F., 1980. Stress profide for teachers: Test mamal preliminary research date. (Departament of Education: California. San Dicgo). 